\title{
La ropa, los adornos y el cuerpo. La interacción entre el productor, el objeto y el consumidor
}

\author{
Clothes, ornaments and body. The interaction between the \\ producer, the object and the consumer
}

NURIA PEIST

\begin{abstract}
Resumen: A partir de dos documentales, se analizan algunos de los valores que los diseñadores y artesanos otorgan a su oficio. Aquellos que se dedican al diseño de indumentaria y adorno son más conscientes del cuerpo del sujeto que consumirá su producción. Los que practican oficios relacionados con el resto de los objetos de uso remarcan sobre todo el contacto físico y emotivo que ellos mismos tienen con lo producido. En ambos casos, se observa una marcada conciencia de la relación de los objetos con los cuerpos de los consumidores, en el primer caso, y de los productores, en el segundo. Esta conciencia permite reflexionar sobre cómo el contacto físico con los objetos colabora a las relaciones entre las personas. Asimismo, ayuda a analizar cómo la explotación en el trabajo y la excesiva valoración de los aspectos formales de los sujetos y los objetos producen un alejamiento de lo corporal y una ruptura de las relaciones.
\end{abstract}

Palabras clave: Cuerpo, diseño, artesanía, indumentaria, adorno, sociología de la moda.

\begin{abstract}
Some of the values that designers and craftsmen give to their craft are analyzed based on two documentaries. Those engaged in clothing design are more aware of the consumer's body. Those who practice trades related to other objects of use highlight the physical and emotional contact that they have with their production. In both cases, there is a strong awareness of the relationship between the objects and the bodies, of the consumers in the first case, and producers in the second. This awareness reflects on how the physical contact with objects collaborates in the relationship between people.

It also helps analyze how labour exploitation and the overvaluation of the formal aspects of the subjects and objects produce estrangement with the body and a disruption in relations.
\end{abstract}

Keywords: Body, design, crafts, clothing, ornaments, fashion sociology.

Fecha de recepción: 10/06/2016. Fecha de aceptación: 06/09/2016.

* Universidad de Barcelona -Profesora lectora- nuriapeist@ub.edu Líneas de investigación: historia y filosofía del arte moderno y contemporáneo, sociología del arte. Publicaciones recientes: "Las obras de arte, las personas y los materiales. Un análisis fenomenológico y sistémico del arte actual”, en Artnodes N $\mathrm{N}^{\mathrm{o}}$ 15, pp. 15-21, 2015. "Historia del arte, estudios visuales y sociología del arte: un debate ideológico-disciplinar", en Millcayac. Anuario de la Facultad de Ciencias Políticas y Sociales de la Universidad de Cuyo. Dossier Sociología del arte, vol. 1, n 1 , pp. 31-48, 2014. 
El desarrollo de la producción, la circulación y el consumo de objetos, mensajes y espacios en forma de bienes y servicios marca el ritmo de nuestra cultura actual. Mucho se ha dicho sobre la fetichización de los objetos fruto del capitalismo multinacional: junto con el consumo desmedido, la superficie de las cosas y de los cuerpos se impone sobre la necesidad de uso y sobre la identificación de los sujetos y sus relaciones (Jameson, 1996). No obstante, lo material, la identidad y la comunicación siguen teniendo relevancia tanto en la producción como en el consumo. A veces de forma constructiva, como en la relación entre las personas y los materiales, a veces de forma destructiva, como sucede en las relaciones de explotación de los cuerpos que viven y trabajan. Prestar atención al ámbito de la producción de objetos puede colaborar a comprender algunas de sus especificidades y parte de su sistema de relaciones.

En el ámbito del diseño, existen distintas ramas de especialización que siguen operativas en ámbitos académicos y profesionales: diseño industrial o de producto, diseño espacial, diseño gráfico y diseño de moda o de indumentaria. Esta división refleja las diferencias que existen cuando se piensa o proyecta un espacio, un mensaje o un objeto. Dentro de este orden, llama la atención el alto grado de autonomía del entorno de la ropa y del adorno: productos industriales que bien podrían incluirse en el ámbito más amplio del diseño industrial o de producto y sin embargo tienen su propio espacio y especialización. Y esta será nuestra pregunta y excusa inicial, ¿qué particularidades posee la producción de ropa y de adorno frente otros productos artesanales o industriales?

Por un lado, recordemos la manera en que los espacios públicos de la modernidad se transformaron en grandes escenarios de presentación frente a los otros (Sennett, 1977). En el tumulto de la multitud, los encuentros suelen ser fugaces y la apariencia es la carta de presentación. La imagen forma una parte importante en la constitución de lo que somos y, sobre todo, de lo que queremos ser (Goffman, 2004). La ropa, el adorno y las maneras en que se presenta el cuerpo se transformaron en útiles identitarios que pusieron todo lo relativo al aspecto en el foco de atención. Por otro lado, desde una perspectiva fenomenológica, la ropa y el adorno tienen una relación estrecha con el cuerpo. Hay autores que defienden que ropa, cuerpo e identidad social son indisociables y forman parte de una misma realidad (Entwistle, 2002). Considerando esta doble perspectiva, se comprende que el sistema de la moda haya desarrollado un espacio propio, con sus agentes e instituciones, para responder a un mercado de la apariencia cada vez más complejo.

Para profundizar en este terreno, estudiaremos los objetos que nos rodean y los objetos que nos visten y nos adornan para comprobar si existen especificidades y puntos de contacto susceptibles de análisis. Observaremos también los sistemas de relaciones entre las cosas y las personas que las producen y las consumen. Nos centraremos en los objetos materiales, en la producción y no en la reproducción, como apunta Jameson (1996, 83), para analizar lo tangible, la elaboración y el uso material de las cosas y su relación con el cuerpo.

Con tal fin, analizaremos los discursos de un grupo de artesanos y diseñadores que fueron entrevistados para una serie de tres videos realizados por Jesús-Àngel Prieto bajo el encargo del Consorcio de Comercio, Artesanía y Moda de la Generalitat de Cataluña. Sólo analizaremos dos de ellos, cuyos títulos significativos son $O$ de objeto (2009) y $S$ de sujeto (2010). En el análisis se tendrá en cuenta que el director posee una visión personal, sobre todo en los criterios de selección de los entrevistados y en la organización y estructura del 
tema. Pero aunque la suya sea una mirada de especialista, las declaraciones de los artesanos y diseñadores nos ayudarán a comprender algunas de las valoraciones que los actores aportan a cada ámbito especifico.

\section{El objeto y el productor}

En el documental $O$ de objeto, centrado en la producción de piezas sobre todo artesanales, se observa la relación que los artesanos y los diseñadores mantienen con el objeto producido. No existe en los discursos una conciencia de las personas que utilizarán los objetos. En su lugar, se observa la importancia que posee la relación afectiva entre el productor y su producto, con un énfasis especial puesto en la materia:

El violín es el objeto más maravilloso creado en madera.

Es el mismo material el que te dicta hacia dónde has de ir (...) las yemas de tus dedos son las que te hacen sentir, las que te hacen escuchar el ruido.

Hay que darle un juego más amable a la piedra, sin ángulos rectos.

Dentro de la materia hay información que es importante y que está oculta.

Por otro lado, existe una conciencia de la manera en que el acto productivo conforma la identidad personal y profesional:

El objeto no es solo una interface mecánica entre nosotros y el mundo, sino también una manera de explicarnos a nosotros mismos.

Nuestro trabajo nos sirve para construirnos a nosotros mismos.

Yo creo que el oficio se lleva dentro.

En las declaraciones, observamos la importancia que se le otorga al contacto físico con el producto: la materia dicta hacia donde hay que ir, hay que escucharla, sentirla, moldearla. Dicha lógica proviene del ámbito de lo artesanal, en donde el tiempo dedicado y el contacto directo con el material aportan una calidad sensible y emocional al acto productivo. También vemos que la producción se percibe como constitutiva de la identidad. En algunos casos, no existe la conciencia de la presencia de un afecto o de la constitución de uno mismo, sino que se explica la utilidad de un objeto, en este caso, de uso colectivo, tal y como refiere un guardabosque:

Esto es fácil. Una cesta sí que tiene trabajo, te lleva un día de trabajo. El nombre de esto, aquí le decimos besulla. La besulla para beber. Y esto normalmente lo hacían los pastores. Antes había muchos pastores aquí, hacia la montaña. Y en cada fuente tenían una colgada, en una rama. Y para beber, para beber agua, mira, todos bebían de la misma cosa.

Esta diferencia en la manera de valorar el trabajo artesanal puede estar motivada por la posesión de una cultura específica de la emoción y la subjetividad. En el caso del guardabosque, la valoración del objeto es igual de importante, pero no se identifican emociones asociadas a la relación con la materia. 
No obstante, en ambos casos existe una conciencia del contacto con el objeto, algo que se pierde cuando interviene el proceso industrial. La complejidad del trabajo mecanizado trajo como consecuencia la división y especialización del trabajo. Los diseñadores proyectan la forma de los objetos y los trabajadores fabrican el producto operando máquinas o manipulando materiales de forma mecánica la mayoría de las veces. La distancia se agudiza al intervenir las relaciones de explotación en el trabajo: condiciones de esclavismo extremas que posibilitan la multiplicación de objetos de consumo incrementando la distancia entre el productor y el consumidor que se transforma en una suerte de cómplice inconsciente. En el documental se observa cierta conciencia de esa distancia fría entre el productor y el consumidor:

Una pieza que llegues a querer tanto que no puedas prescindir de ella y que no sepas quién la ha hecho (...) Te gustaría abrazarlo y decirle que me has provocado una sensación, que te felicito.

En el todo a 100 (...) hay una pérdida de relación entre el objeto y la persona que lo ha hecho.

Mientras que teóricos del diseño como Guy Julier (2010) defienden el oficio como una práctica relacional compleja, como un acto comunicativo y plantean la necesidad de hablar de una "cultura del diseño", en las prácticas reales de producción y consumo se profundiza la ruptura de los vínculos: entre el productor y el producto y entre el productor y el consumidor.

\section{El objeto y el cuerpo}

En el documental $S$ de sujeto, es llamativa la conciencia que los artesanos y diseñadores tienen respecto al cuerpo que usará los objetos que producen:

Las joyas nacen como una necesidad mía, propia. Después de esta reflexión pienso en la persona que llevará la joya.

Algunas piezas mías, por ellas solas, no pueden decir nada. Son un trozo de metal sobre la mesa. Necesitan de la combinación de un cuerpo (...), que haya una relación, que a una persona le ayude a manifestarse.

A cada persona se le tiene que poner una pieza adecuada a su problema.

Las piezas tienen una historia, tienes que conocer a las personas, por ejemplo, en la ropa de mi abuela hay una parte de ella.

A diferencia de otros objetos de uso, la ropa y los adornos se relacionan de manera muy estrecha con el sujeto consumidor. Los productores toman conciencia del acto comunicativo desde el momento de su elaboración. Además, son conscientes de que ayudan al sujeto a volver su cuerpo social, "a manifestarse", a adecuarse a "su problema". El contacto entre el objeto y el sujeto que lo porta es tan estrecho que se vuelven una misma entidad y, como consecuencia, el vinculo entre productor y consumidor se intensifica a la vez que se intensifican los lazos con el mundo que los rodea: 
Lo que hago que se pueda utilizar, que tenga un interés (...) que pueda tener una parte de complicidad con la persona que lo lleve. Para sentirse mejor, para que alguno le diga cosas, para crear una interrelación con las otras personas.

Estoy siempre intentando imaginar lo que siente esa persona en el momento en que se la deposite, se la ponga sobre sí mismo, qué efectos provocará con él.

A partir del vestir se pueden decir muchas cosas. Podemos decir muchas cosas de nuestra personalidad que no yendo desnudos.

La ropa (...), cada uno le da un lenguaje diferente.

Este acto comunicativo se llevará a cabo si el productor puede pensar y mantener un contacto estrecho con el objeto que produce, si no, como mencionamos en el apartado anterior, el objeto producido industrialmente o en condiciones de explotación estará ligado en mayor o menor medida al cuerpo del sujeto, pero su relación con el ámbito de la producción se perderá.

En el documental, también vemos que el objeto en contacto con el cuerpo puede tomar vida, corporizarse, en palabras de Merleau-Ponty, a través de una mirada táctil y orgánica sobre ellas: "la mirada misma las envuelve, las viste con su carne" como afirma el autor en Lo visible y lo invisible $(1970,164)$. Así lo perciben algunos entrevistados:

Lo que me interesa es que tenga su función (...) Aparte de esto, a mí lo que me interesa mucho es que las piezas que estamos haciendo, de alguna manera, tengan su propia vida, cuando va pasando el tiempo, van envejeciendo, el propio uso de la persona le va dando una forma, le va dando un carácter. Al final esa pieza (...) acaba pues prácticamente siendo otra cosa de lo que yo había hecho.

Es una pieza que tiene que ser portable al $100 \%$ (...), una pieza que con el tiempo va cogiendo su vida. Tu manera de ser se transfiere a esta piel, la piel lo absorbe todo.

En el polo opuesto, tal y como veremos en el siguiente apartado, los objetos pueden alejarse del acto comunicativo. En tal caso, la valoración se acerca a la formalidad, el objeto se aprecia por sus cualidades estéticas y se produce un alejamiento de la mayoría de los consumidores, que deben tener una cultura específica para poder valorarlo más allá de la experiencia funcional.

\section{El objeto escultura}

Las relaciones que existen entre el mundo del diseño y el mundo del arte son numerosas y variables. A menudo, se intenta cargar los objetos, mensajes y espacios de uso cotidiano de valores asociados a lo estético: innovación, originalidad, belleza, creatividad, etc. Los objetos de consumo tienen un valor estético pero siempre están en contacto con otro tipo de factores: el funcional, el tecnológico, el económico y, sobre todo, el comunicativo. Conforme el diseño de los objetos se acerca más a la valoración formal y se desprende de los otros factores, pierde su efectividad de uso y la comunicación cambia de tono:

Y es un mortero simplemente. Es un mortero que prácticamente es una escultura. 
Los objetos, cuando pierden el sentido utilitario, tienen el valor de objetos estéticos tan interesantes y tan bonitos como puede ser una escultura. Son esculturas (...) y en algún momento sirvieron para poner aceite, agua, grano.

[La cerámica en la década de 1950] la gente la compraba, la coleccionaba, iba a exposiciones (...) Miró abrió posibilidades en el mundo de la cerámica.

En las declaraciones, los objetos parecen dignificarse, volviéndose interesantes en cuanto adquieren connotaciones escultóricas. Pero un objeto solo valorado por sus características formales pierde contacto con el uso y con el cuerpo de las personas, dignificándose en una suerte de distancia estética impersonal. Lo valorado como artístico se percibe del lado de la inspiración repentina, mientras que en el otro extremo el diseño de un objeto de uso se caracteriza por la lentitud, el trabajo, la dedicación y el proyecto:

El arte es fruto de un momento, ¡pam!, una chispa. La artesanía tiene esta parte lenta.

Pero en ocasiones el productor manifiesta la necesidad de un objetivo, de una dirección que intensifique la comunicación con las necesidades concretas o, para ser más precisos, de una función social. En este caso, no se reivindica la autonomía creativa, propia del mundo del arte, sino que el encargo se asume como parte del proceso creativo:

Trabajando encuentras un efecto estético que te gusta y dices ostras, esto, qué chulo, pero qué hago con esto. A veces el hecho de que alguno te encargue, eso, un plato, dices ostras, ya está, porque la utilidad ya me la ha dado el otro.

No obstante, afirmar que algo no es artístico, o que no posee las lógicas específicas de la creatividad, parece avivar el fantasma de la falta de calidad y, sobre todo, de lo ilegítimo cultural. Los objetos de diseño o artesanales siempre están peligrosamente asociados al uso cotidiano y siempre hay algo que se les tiene que prestar para conseguir una mayor legitimidad. Sin embargo, potenciar la función social que puede tener un objeto, la manera en que puede ayudar a mejorar la vida de las personas, puede ser un antídoto contra la defensa del objeto de uso como objeto artístico puro, desvinculado de los demás factores que lo configuran, que conforman su especificidad como objeto cultural y que colaboran al acto comunicativo.

\section{El cuerpo escultura y el bienestar interior}

Para completar nuestro acercamiento, en este apartado nos centraremos en las reflexiones de diversos autores que plantearon qué sucede con los cuerpos cuando se transforman en foco exclusivo de atención, se les aplica un modelo único y dominante de apreciación y juicio y se les exige todo lo exigible: el equilibrio modélico exterior, pero también el interior. El vestido y el adorno juegan un papel importante en este tipo de objetivación.

La valoración de las formas alejadas de la funcionalidad y la comunicación con el entorno se manifiesta también en la indumentaria y el adorno. Los desfiles son espacios privilegiados para observar la estetización de la ropa y los complementos: trajes pesados o 
incómodos, modelos con caras tapadas -como en el desfile de la Maison Martin Margielae hipersexualización de los cuerpos. Tal y como nos recuerda Pascal Ory $(2006,147)$, la palabra "bronceado" era propia de las Bellas Artes y designaba un objeto recubierto con una pátina que imita el aspecto del bronce. Después de la Primera Guerra Mundial, pasa a utilizarse para designar un color de los cuerpos que colabora a mejorar la apariencia.

La metáfora es muy sugestiva. No solo los objetos pueden apreciarse por sus valores escultóricos, sino que también los cuerpos pasan a concebirse como susceptibles de ser moldeados, "pedagogicamente disponibles" para transformarse en capital, tal y como afirma José Luis Moreno Pestaña (2016, 9-44). En este caso, los valores formales transforman el cuerpo en un objeto también, tapado, bronceado, cubierto por un modelo único y universal que se deposita en los cuerpos e impide la dialéctica que nos constituye como personas a partir de la comunicación con los demás.

La valoración formal, escultórica, modélica del cuerpo-objeto remite al concepto de perfección, de un único cuerpo posible y armónico. Un cuerpo hipervalorado, con una atención excesiva sobre él. Esta constante preocupación incluso médica por el cuerpo parece acercarnos más a quienes somos pero, tal y como afirman algunos autores, nos alejan de nosotros y de los demás. En el libro Moral corporal, trastornos alimentarios y clase social, José Luis Moreno Pestaña analiza las fases de un trastorno: la excesiva atención a una parte de la existencia -en este caso en forma de tensión corporal- provoca una falta de cuidado de otros ámbitos. Su correlato es una destrucción de los "marcos de experiencia compartidos" $(2010,55)$.

Respecto al exceso de atención sobre lo físico, Anne Marie Sohn en "El cuerpo sexuado" observa el retroceso del pudor que provoca un cuerpo "exhibido, omnipresente en el espacio visual" $(2006,101)$. Michèle Pages-Delon (1989) nos habla del "capital de apariencia", y podemos agregar que, sobre todo a lo largo del siglo XX, se produce una intensa corporización de ese capital, sobre todo en la imagen femenina. En el terreno artístico, vemos traducida esta preocupación constante por lo corporal en las visiones de cuerpos mutilados y destrozados, desde Antonin Artaud y los surrealistas, pasando por Bataille y los casos extremos del Accionismo Vienés. Yves Michaud nos advierte que en muchos casos la atención artística por el cuerpo no posee la carga política de, por ejemplo, la Nueva Objetividad alemana, dejándonos una conciencia por lo corporal vacía y sin un correlato social:

Pero en general, los ultrajes a los que se someten los cuerpos, pasan al arte sin tener una significación política directa: se convierten en ultrajes autoinflingidos en el marco de rituales religiosos o a título de afirmaciones de existencia (Michaud, 2006, 409).

Pero el concepto de perfección asociado a un cuerpo demasiado atento de sí mismo no es solamente externo. Tal y como afirma George Vigarello, en la segunda mitad del siglo XX se desarrolla una obsesión por la perfección interior, por la consecución del equilibrio en todos los ámbitos, algo que comúnmente se denomina bienestar y que parece garantizarnos la llave a la belleza, un reclamo que se utiliza incluso en la publicidad cosmética: si te sientes bien contigo misma, alcanzarás la belleza, en una mezcla de imagen exterior y paz interior. Pero 
el autor advierte que esta exigencia aporta más crispación que tranquilidad. Nada puede ir mal, todo tiene que estar bajo control, cada uno puede optar por ser bello. Democratización de la belleza y máxima exigencia personal, cualquier fallo puede frustrar el proyecto total:

Indudablemente, la exigencia de la belleza se ha fortalecido hoy: cuerpo más expuesto, identidad más 'corporizada'. Pero al democratizarse, al diluirse sin fronteras, al prometer solamente el bienestar, también ha fabricado, de manera muy conjunta, la distensión y la crispación (Vigarello, 2005, 258).

A lo largo de nuestro análisis, vimos que una de las características que permite precisar la particularidad de la indumentaria y el adorno es su contacto con el cuerpo. Los diseñadores y artesanos entrevistados en los documentales muestran una conciencia llamativa respecto a las personas que usarán sus objetos, algo que no ocurre con otro tipo de oficio. Era nuestra hipótesis de partida. Pero cuando observamos el ámbito de la producción de los demás objetos de uso, nos dimos cuenta que existe otra relación fundamental presente en el discurso de los artesanos: la del objeto producido en contacto con el cuerpo del productor. Vemos también que cuando estas relaciones físicas se rompen, sea por la aplicación de modelos que no dejan desarrollar las potencias corporales propias, en el primer caso, o por las actuales condiciones de sobre explotación en el segundo, lo que se resienten son las relaciones entre las personas. Las relaciones entre el productor y el consumidor, una vez perdida la relación entre materia, producción, distribución y consumo local, y alcanzadas unas relaciones de esclavitud en las fuerzas de trabajo que nos obligan a una distancia ética deshumanizada. Las relaciones también entre los cuerpos y las relaciones que establecemos entre ellos, los placeres físicos compartidos, los contextos de interacción, que, tal y como indican los autores mencionados, necesitan cuerpos que acompañen y no cuerpos que se piensan de manera constante a sí mismos.

También vimos que algunos de los artesanos y diseñadores diferencian muy bien entre lo que se considera arte y lo que es la artesanía, un proceso este último que implica un esfuerzo y una conciencia imprescindible respecto a su función social. La valoración estética de los objetos de uso cotidiano, muchas veces denominada valor añadido y dedicada a aportar un plus publicitario al objeto mismo (Sparke, 2010, 79-81), no es de ninguna manera un impedimento para el desarrollo pleno de su función. No obstante, la exigencia de un valor artístico per se, desvinculada de la apreciación de su valor social, conduce también a una ruptura con las relaciones y con la comunicación con la mayoría de las personas. Pierre Bourdieu en su libro Un arte medio. Ensayo sobre los usos sociales de la fotografía (2003), explica que la invención de la fotografía tuvo tanto éxito porque ayudó a potenciar funciones sociales preexistentes a su aparición: la solemnización de los momentos compartidos. Nos advierte que el valor puramente estético se presenta sobre todo en espacios sociales en donde los lazos con la comunidad son más débiles. Y a modo de reflexión final, es posible plantear que el valor estético debe acompañar, pero es problemático que se transforme en el único criterio de valor, en un modelo único de legitimidad cultural. En su lugar, objetos valorados también por su función, fabricados para y en contacto con la gente, respetando a quien lo usa y a quien lo produce. 


\section{Bibliografía}

Bourdieu, Pierre (2003): Un arte medio. Ensayo sobre los usos sociales de la fotografía, Barcelona, Gustavo Gili, Barcelona.

Entwistle, Joanne (2002): El cuerpo y la moda. Una visión sociológica, Paidós, Barcelona.

Goffman, Erving (2004): La presentación de la persona en la vida cotidiana, Amorrortu, Buenos Aires.

Jameson, Fredric (1996): Teoría de la postmodernidad, Trotta, Madrid.

Julier, Guy (2015): La cultura del diseño, Gustavo Gili, Barcelona.

Merleau-Ponty, Maurice (1970): Lo visible y lo invisible, Seix Barral, Barcelona.

Michaud, Yves (2006): "Visualizaciones. El cuerpo y las artes visuales", en Corbin, A., Courtine, E., Vigarello, G. (Eds.), Historia del cuerpo III. Las mutaciones de las miradas. El siglo XX, Taurus, Madrid, pp. 401-417.

Moreno Pestaña, José Luis (2016): La cara oscura del capital erótico. Capitalización del cuerpo y trastornos alimentarios, Akal, Madrid.

Moreno Pestaña, José Luis (2010): Moral corporal, trastornos alimentarios y clase social, Centro de Investigaciones Sociológicas, Madrid.

Ory, Pascal (2006): "El cuerpo ordinario", en Corbin, A., Courtine, E., Vigarello, G. (Eds.), Historia del cuerpo III. Las mutaciones de las miradas. El siglo XX, Taurus, Madrid, pp. 135-164.

Pages-Delon, M. Michèle (1989): Le corps et ses apparences. L'envers du look, L'Harmattan, París.

Prieto, Jesús-Angel (2010): $S$ de subjecte (A d'artesania) [Archivo de vídeo] Recuperado de https://vimeo.com/59338580

Prieto, Jesús-Angel (2009): O d'objecte (A d'artesania) [Archivo de vídeo] Recuperado de https://vimeo.com/58787869

Sennett, Richard (1978): El declive del hombre público, Península, Barcelona.

Sohn, Anne-Marie. (2006): "El cuerpo sexuado", en Corbin, A., Courtine, E., Vigarello, G. (Eds.), Historia del cuerpo III. Las mutaciones de las miradas. El siglo XX, Taurus, Madrid, pp. 101-107.

Sparke, Penny (2010): Diseño y cultura. Una introducción. Desde 1900 hasta la actualidad, Gustavo Gili, Barcelona.

Vigarello, Georges (2005): Historia de la belleza. El cuerpo y el arte de embellecer desde el Renacimiento hasta nuestros días, Nueva Visión, Buenos Aires. 
